\title{
A novel method of ESR oscillating magnetization value determination in strongly correlated metals
}

\author{
Marat Gilmanov ${ }^{1,2, *}$, Alexey Semeno ${ }^{2}$, Alexander Samarin ${ }^{2}$, and Sergey Demishev ${ }^{1,3}$. \\ ${ }^{1}$ Moscow Institute of Physics and Technology, Dolgoprudny, Russia \\ ${ }^{2}$ Prokhorov General Physics Institute of RAS, Moscow, Russia \\ ${ }^{3}$ National Research University "Higher School of Economics", Moscow, Russia
}

\begin{abstract}
We propose a powerful method of direct measurement of oscillating magnetization by the electron spin resonance, based on dependence of resonant conditions on geometry of the experiment. Theoretical consideration of the matter leads to a simple expression for oscillating magnetization. Approbation of this method is implemented by means of cavity ESR spectrometer $(60 \mathrm{GHz})$ on two diverse metallic systems, where static magnetization at the resonance field varies by an order of magnitude. Quantitative values of oscillating magnetization ( $905 \mathrm{G}$ for $\mathrm{EuB}_{6}$ at $\mathrm{T}=4.2 \mathrm{~K}$ and $94 \mathrm{G}$ for $\mathrm{CeB}_{6}$ at $\mathrm{T}=1.8 \mathrm{~K}$ ) are in appropriate agreement with the one obtained by the other methods.
\end{abstract}

\section{Introduction}

It was noticed quite some time ago that application of the concept of the permeability coefficient could not explain all experimental data concerning dynamic magnetic properties and permeability tensor should be used [1]. However, in experiment one could observe only the scalar values and therefore the correspondence between tensor and scalar quantities is not trivial, as it was demonstrated by Young and Uehling for two main cases of magnetization parallel and perpendicular to the surface of a metallic sample [2].

The parameters describing the electron spin resonance (ESR) line shape give information about magnetic state of the material, and while g-factor and line width could be directly obtained from the experiment, the integrated intensity of the resonance line is believed to be proportional to the static susceptibility of oscillating moments and requires significant additional efforts to be obtained in absolute values from the ESR data. Basically, the integrated intensity is defined by oscillating magnetization $\mathrm{M}_{0}$, which may be introduced in the same way as in [3,4]. The $\mathrm{M}_{0}$ parameter defines magnitude of the ESR absorption, and in most cases it coincides with static magnetization $\mathrm{M}_{\mathrm{st}}$ $\left(\mathrm{EuB}_{6}[3], \mathrm{YbRh}_{2} \mathrm{Si}_{2}[5]\right)$, but there are some materials $\left(\mathrm{CeB}_{6}[4],[6]\right)$ that demonstrate a significant difference between the oscillating $\mathrm{M}_{0}$ and total static magnetization $\mathrm{M}_{\mathrm{st}}$ measured by standard technique for studying of magnetization. The comparison of $\mathrm{M}_{0}$ and $\mathrm{M}_{\mathrm{st}}$ may provide important information on different magnetic contributions or interactions within the system [6].

In addition, the problem of experimental determination of $\mathrm{M}_{0}$ recently arose due to growing interest in strongly correlated metallic systems [4-8].
However, only a few methods have been developed in this area up to now.

One of the techniques that could be used to obtain $\mathrm{M}_{0}$ is quite similar to the one being applied often to the dielectric samples. It consists in measuring the investigating material together with some reference sample with known magnetic properties and magnetic resonance response in similar conditions. Such procedure was performed for the heavy fermion compound $\mathrm{YbRh}_{2} \mathrm{Si}_{2}$ by comparison the ESR intensity with the samples $\mathrm{YPd}_{3}: \mathrm{Yb} 1 \%$ [5] and $\mathrm{YPd}_{3}: \mathrm{Yb} 0.6 \%$ [7], and it was discovered that $\mathrm{M}_{0}$ and $\mathrm{M}_{\mathrm{st}}$ in this system coincides. Nevertheless, this method has some major uncertainties which are due to (i) the assumption concerning comparable quality factors of the microwave cavity for both samples and from (ii) uncertainties in determining the sample size, mass, and penetration depth [5].

Another method was developed for the ferromagnetic strongly correlated metal $\mathrm{EuB}_{6}$ [3] and been also applied to a helimagnet compound $\mathrm{MnSi}$ [8] and dense Kondo system $\mathrm{CeB}_{6}$ [4]. This method exploits the fact that the absorption in the sample depends on the combination $\left(\mu_{\text {eff }} \rho\right)^{1 / 2}$ of effective permeability $\mu_{\text {eff }}$ and resistivity $\rho$. In the case of strong magnetoresistance, the dependence of resistivity on the magnetic field $\rho(\mathrm{H})$ could be used to calibrate values of $\mu$ in the absolute values. Application of this method to the $\mathrm{CeB}_{6}$ system showed a significant deviation of $\mathrm{M}_{0}$ from $\mathrm{M}_{\mathrm{st}}$, which is a consequence of the key role of ferromagnetic correlations in the genesis of magnetic resonance in dense Kondo systems [4]. However, the main difficulty of this method is that it requires strong dependencies of magnetoresistance of investigated material to provide acceptable accuracy of absolute calibration of $\mu_{\text {eff. }}$ 
We argue that the same parameter $\mathrm{M}_{0}$ could be directly obtained from the difference in positions of the ESR line in experiments with different mutual orientations of magnetization with relation to the sample surface. Testing of this method was performed for two different systems with high $\left(\mathrm{EuB}_{6}\right)$ and low $\left(\mathrm{CeB}_{6}\right)$ oscillating magnetization to demonstrate a wide range of the applicability of proposed ansatz.

\section{Method}

\subsection{Theoretical background}

As it was mentioned above, the effective resonant function of magnetic permeability $\mu_{\text {eff }}(H)$ includes two elements of permeability tensor $\mu(H)$ and $\mu_{\alpha}(H)$ [2] due to gyrotropic origin of ESR. While in the case $\mathbf{k} \| \mathbf{H}$ ESR response is defined by two circularly polarized functions $\mu_{ \pm}=\mu \pm \mu_{\alpha}$, the scheme with $\mathbf{k} \perp \mathbf{H}$ results in $\mu_{\text {eff }}=\mu-\mu_{\alpha}{ }^{2} / \mu$ [2]. We wish to emphasize that in these two cases the parameters of the ESR line should be strongly affected not only by the demagnetization fields, but also by the conditions of the experiment itself.

Exact equations describing the ESR line shape in these two cases could be found elsewhere [2]. For current consideration, we only would be interested in the difference of resonant conditions.

For the case of $\mathbf{k} \perp \mathbf{H}$, the position of resonance $\mathrm{H}_{\perp}$ could be described by:

$$
\omega_{0}^{2}=\gamma^{2} H_{\perp}\left(H_{\perp}+4 \pi M_{0}\right)\left(1+a^{2}\right)
$$

and

$$
\omega_{0}=\gamma H_{\|}
$$

for the case of $\mathbf{k} \| \mathbf{H}$. Here $\mathrm{H}_{\perp}$ and $\mathrm{H}_{\|}$correspond to resonant fields in considered experimental geometries and $\alpha$ stands for dissipation parameter in Landau-Lifshitz damping term.

Although the equations (1)-(2) depend on $\mathrm{M}_{0}$ only, the influence of the demagnetization fields should also be taken into account. This could be performed quite easily by modification of values of the external magnetic field for both cases as $\mathrm{H}_{\|}{ }^{\prime}=\mathrm{H}_{\|}-\mathrm{N}_{\mathrm{z1}} \mathrm{M}_{\mathrm{st}}$ and $\mathrm{H}_{\perp}{ }^{\prime}=\mathrm{H}_{\perp}-$ $\mathrm{N}_{\mathrm{z} 2} \mathrm{M}_{\mathrm{st}}$ where $\mathrm{N}_{\mathrm{z} 1}$ and $\mathrm{N}_{\mathrm{z} 2}$ denote the demagnetization factors for different directions of the magnetic field with respect to the sample. Such correction of the $\mathrm{H}_{\perp}$ and $\mathrm{H}_{\|}$ should be performed precise enough for accounting of the significant impact of the $\mathrm{N}_{\mathrm{z} 1}, \mathrm{~N}_{\mathrm{z} 2}$ on the final result. This could be done by means of numerical simulation applicable to any particular form of the sample [9]. Values of $\mathrm{N}_{\mathrm{z} 1}$ and $\mathrm{N}_{\mathrm{z} 2}$ calculated in this way for disk shaped samples are given in Table 1 . It also should be noted that accounting for the demagnetization factor would not lead to Kittel resonant conditions [10] due to the interaction of the sample with microwave radiation being limited by the skin-effect.

Summing up equations (1), (2) and taking into account that experiments in both geometries are performed at the same frequency, the following expression for oscillating magnetization $\mathrm{M}_{0}$ could be derived:

$$
4 \pi M_{0}=\frac{\left(H_{\|}-N_{Z 1} M_{s t}\right)^{2}}{\left(H_{\perp}-N_{Z 2} M_{s t}\right)\left(1+a^{2}\right)}-\left(H_{\perp}-N_{Z 2} M_{s t}\right)
$$

\subsection{Experimental details}

Experimental method was tested with the help of high frequency $(60 \mathrm{GHz})$ cavity ESR spectrometer based on Agilent E8261C Network Analyzer [11]. Sample is placed as a part of the cavity wall to evade field inhomogeneity effects. Two types of coupling between waveguides and resonator are used to establish different geometries of the experiment (Fig.1). Also, cavities are designed to be practically identical in both experiments to simplify the calculations.

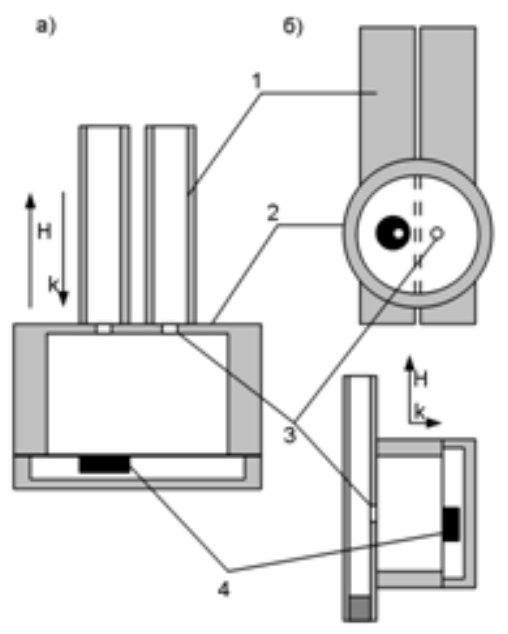

Figure 1. Experimental layouts corresponding to the cases of $\mathbf{k} \perp \mathbf{H}$ (a) and $\mathbf{k} \| \mathbf{H}$ (b) in ESR experiment. The direction of the vector $\mathbf{k}$ corresponds to its orientation at the sample surface. 1 -waveguides, 2 -cylindrical resonator, 3 communication holes, 4 - sample on thin cupper foil aperture.

\section{Approbation}

Two rather different metallic systems are selected to demonstrate a wide range of applicability of the proposed method. First, $\mathrm{EuB}_{6}$ is a ferromagnetic strongly correlated metal with $\mathrm{M}_{\mathrm{st}}=912 \mathrm{G}$ at resonant field (31.6 kOe). Spectra obtained at $\mathrm{T}=4.2 \mathrm{~K}$ for both magnetization conditions are presented at Fig.2. Narrow ESR line and a large magnetization leads to a significant difference in line shape and resonance fields as well as to considerable values of demagnetization fields. The obtained parameters are presented at the Table 1 . The calculated value of $\mathrm{M}_{0}$ coincides with the one obtained by different method [3] and, as expected, with the value of static magnetization within the error limits.

Dense Kondo system $\mathrm{CeB}_{6}$ is on the other edge of experimental complexity. Small values of magnetization $\left(\mathrm{M}_{\mathrm{st}}=96 \mathrm{G}\right.$ at $\left.\mathrm{T}=1.8 \mathrm{~K}\right)$ cause the difference in resonant fields to be relatively low (see Fig.3). Moreover 
widening of the ESR line makes more difficult the resonance field determination. Nevertheless, even in this case, the calculated $\mathrm{M}_{0}$ values agree well with the ones reported earlier [4] (see Table 1).

For the case of differing $\mathrm{M}_{0}$ and $\mathrm{M}_{\mathrm{st}}$ in $\mathrm{CeB}_{6}$ $(\mathrm{T}=2.6 \mathrm{~K})$, considerable broadening simultaneously with decreasing in intensity of the line prevents the possibility of obtaining very accurate value of $\mathrm{M}_{0}$. In spite of that it is still possible to obtain estimate the value of $\mathrm{M}_{0}$ being significantly lower than $\mathrm{M}_{\text {st }}$ (Table 1) which also correlates with the results obtained earlier [6].

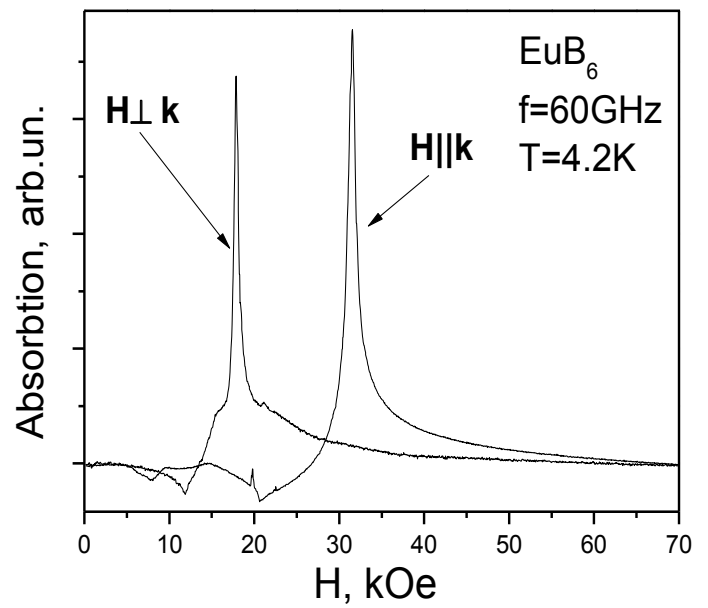

Figure 2. ESR spectra of $\mathrm{EuB}_{6}$ system for two different conditions of magnetization

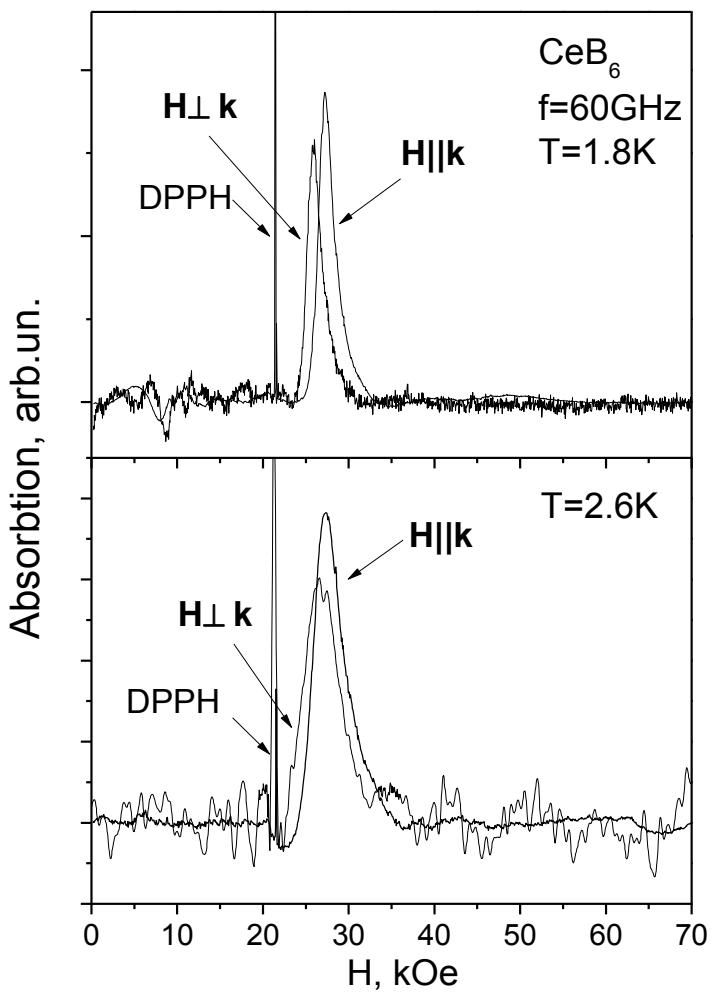

Figure 3. ESR spectra of $\mathrm{CeB}_{6}$ system for two different temperatures and conditions of magnetization. DPPH is a reference signal from an $\alpha$-diphenyl- $\beta$-picrylhydrazyl with $\mathrm{g}$-factor $=2.0036$.
Table 1. Values of demagnetization factors $\mathrm{N}_{\mathrm{z} 1}, \mathrm{~N}_{\mathrm{z} 2}$ calculated by the method described in [9], resonance fields $\mathrm{H}_{\perp}$, $\mathrm{H}_{\|}$and static magnetization $\mathrm{M}_{\text {st }}$ measured in experiments, and oscillating magnetization $\mathrm{M}_{0}$ values estimated from (3).

\begin{tabular}{|c|c|c|c|c|c|c|c|}
\hline Sample & $\begin{array}{c}\mathrm{T}, \\
\mathrm{K}\end{array}$ & $\begin{array}{c}\mathrm{N}_{\mathrm{Z} 1}, \\
4 \pi\end{array}$ & $\begin{array}{c}\mathrm{N}_{\mathrm{Z} 2}, \\
4 \pi\end{array}$ & $\begin{array}{c}\mathrm{H}_{\perp}, \\
\mathrm{kOe}\end{array}$ & $\begin{array}{c}\mathrm{H}_{\|}, \\
\mathrm{kOe}\end{array}$ & $\begin{array}{c}\mathrm{M}_{\mathrm{st}}, \\
\mathrm{G}\end{array}$ & $\begin{array}{c}\mathrm{M}_{0}, \\
\mathrm{G}\end{array}$ \\
\hline $\mathrm{EuB}_{6}$ & 4.2 & 0.86 & 0.07 & 17.6 & 31.6 & 911 & $905 \pm 16$ \\
\hline $\mathrm{CeB}_{6}$ & 1.8 & 0.84 & 0.08 & 25.7 & 27.2 & 96 & $94 \pm 7$ \\
\hline $\mathrm{CeB}_{6}$ & 2.6 & 0.84 & 0.08 & 26.3 & 27.1 & 71 & $21 \pm 10$ \\
\hline
\end{tabular}

\section{Conclusion}

In conclusion, we propose a method of direct measurement of ESR oscillating magnetization $\mathrm{M}_{0}$ based on difference in resonant conditions for different cases of magnetization. It is shown that precise correction on demagnetization fields is required. Method approbation is performed on two rather distinct metallic systems, where static magnetization at the resonance field varies by an order of magnitude. The suggested technique gives appropriate results in agreement with the data collected by the other methods. The obtained results for $\mathrm{CeB}_{6}$ system at temperature $\mathrm{T}=2.6 \mathrm{~K}$ allows concluding that the proposed method could be applied to collect relevant information about oscillating magnetization $\mathrm{M}_{0}$ deviating from the static magnetization $\mathrm{M}_{\mathrm{st}}$.

Acknowledgements. This work was supported by the Russian Scientific Foundation, Grant №17-12-01426

\section{References}

1. J.A. Young jr., E.A. Uehling, Phys. Rev. 90, 990 (1953)

2. J.A. Young jr., E.A. Uehling, Phys. Rev. 94, 3, 545 (1954)

3. A.V. Semeno, V.V. Glushkov, A.V. Bogach, N.E. Sluchanko, A.V. Dukhnenko, V.B. Filipov, N.Yu. Shitsevalova, S.V. Demishev, Phys. Rev. B 79, 014423, (2009)

4. S.V. Demishev, A.V. Semeno, A.V. Bogach, N.A. Samarin, T.V. Ishchenko, V.B. Filipov, N.Yu. Shitsevalova, N.E. Sluchanko, Phys. Rev. B 80, 245106 (2009)

5. J. Sichelschmidt, V.A. Ivanshin, J. Ferstl, C. Geibel, F. Steglich, Phys. Rev. Lett. 91, 156401 (2003)

6. A.V. Semeno, M.I. Gilmanov, A.V. Bogach, V.N. Krasnorussky, A.N. Samarin, N.A. Samarin, N.E. Sluchanko, N.Yu. Shitsevalova, V.B. Filipov, S.V. Demishev, V.V. Glushkov, Sci. Rep. 6, 39196 (2016).

7. J. Wykhoff, J. Sichelschmidt, G. Lapertot, G. Knebel, J. Flouquet, I.I. Fazlishanov, H.A. Krug von Nidda, C. Krellner, C. Geibel, F. Steglich, STAM 8, 389 (2007) 8. S.V. Demishev, A.V. Semeno, A.V. Bogach, V.V. Glushkov, N.E. Sluchanko, N.A. Samarin, A.L. Chernobrovkin, Jetp Lett. 93, 213 (2011) 
9. S. Tandon, M. Beleggia, Y. Zhu, M. De Graef, JMMM 271, 1, 27 (2004)

10. C. Kittel, Phys. Rev. 73, 155 (1948)

11. A.N. Samarin, A.V. Semeno, M.I. Gilmanov,

V.V. Glushkov, I.I. Lobanova, N.A. Samarin,

I.I. Sannikov, N.M. Chubova, V.A. Dyadkin,

S.V.Grigoriev, S.V. Demishev, Phys. Procedia 71, 337 (2015). 